\title{
Evaluation of Zhoushan Maritime Search and Rescue Capability Based on Fuzzy Comprehensive Evaluation
}

\author{
Liang Liu, Zhiliang Li and Hu Liu \\ Port and Transportation College of Zhejiang Ocean University, Zhoushan 316000, P. R. China
}

\begin{abstract}
So as to quantitatively analyze the Zhoushan maritime search and rescue(SAR) capabilities, evaluation model was set up by the theory of fuzzy comprehensive evaluation, combination with analyze of the process of maritime SAR and summarization of the factors influencing the SAR capabilities. The results are analyzed and suggestions are provided for the SAR of Zhoushan sea area.
\end{abstract}

Keywords - SAR ability; comprehensive evaluation; Zhoushan sea area; fuzzy mathematics

\section{INTRODUCTION}

With the approval of Zhejiang Marine Economic Demonstration Zone and the Port integrated development of Zhoushan and Ningbo, the traffic volume of ships in coastal areas of Zhejiang Province has increased dramatically and the navigational environment has become more and more complex. The driving difficulty is constantly increasing. These factors lead to the continuous increase of ship navigational risks and frequent ship accidents occurrence. According to statistics from the Zhoushan maritime SAR center, 54 water traffic accidents has happened in Zhoushan waters in 2010, 542 persons in distress, and the average traffic accident occurred once in 6 days. which caused tremendous loss of human lives, environment and property losses and became one of the harsh challenges of developing the marine economy in Zhejiang. As a last line of defense in saving lives, property and environmental losses caused by maritime traffic accidents, maritime SAR has a particularly significant economic and social significance in enhancing its decision-making level and enhancing its SAR success rate.

\section{MARITIME SAR PROCESS}

Maritime SAR refers to the maritime SAR center after obtaining maritime distress information, coordinating, organizing and directing SAR forces related to SAR operations. Including early warning, distress alert, verification and confirmation, emergency response, response termination. The six phases are the series of concrete actions taken by the search and rescue system in the process of starting the accident detection and responding to the accident. For some special SAR incidents may not require the implementation of each stage; but for some incidents, a stage of the action may include other stages of the action. As a result, some actions in more than two phases may be carried out simultaneously or alternately, resulting in many factors such as human being, environment, rescue and communications.

\section{BASIC PRINCIPLE OF FUZZY COMPREHENSIVE EVALUATION}

The fuzzy comprehensive evaluation method is a systematic analysis method that uses fuzzy mathematics to analyze and evaluate things with "fuzziness". The advantage of the fuzzy comprehensive evaluation method is that it can comprehensively evaluate the object system involving the fuzzy factors and formally transform the uncertainty into Certainty. At present, fuzzy comprehensive evaluation is widely used in many fields. The main process is as follows:

a) Set up fuzzy comprehensive evaluation factor set; divide factor set $U=\left\{U_{1}, U_{2}, U_{3}, \cdots, U_{m}\right\}$. among them $U_{i}$ $(i=1,2,3, \cdots, m)$ Represents the various influencing factors, with varying degrees of ambiguity.

b) Set up the weighting set of influence factors. Generally speaking, the importance and influence of each factor are different, $U_{i}$ The importance of giving it the appropriate weight $a_{i}(i=1,2,3, \cdots, m)$. The set of weights consists of a set of weight factors, that is, $A=\left\{a_{1}, a_{2}, a_{3}, \cdots, a_{n}\right\}$. among them $a_{i} \quad(i=1,2,3, \cdots, m)$ Must satisfy non-negative and normality: $\sum_{i=1}^{m} a_{i}=1 ; \quad a_{i} \geq 0 \quad(i=(1,2,3, \cdots, n))$.

c) Establish the judgment set; judgment set $V=\left\{V_{1}, V_{2}, V_{3}, \cdots, V_{m}\right\}$ A set of outcomes that may result for the object being judged. The purpose of the comprehensive assessment is to give the best result from the judgment set based on a comprehensive consideration of all the factors.

d) Determination of weights; weight is the object of evaluation by the set of factors and factors to determine the importance of the measure. Weight vector A affects the result of comprehensive evaluation. There are many ways to determine A, this article to determine the expert survey method.

e) determine the level of factor evaluation; evaluation level is based on the actual situation to determine the various factors of rating, comments, scale scores. Divide the scale into $\mathrm{H}$ levels, ie $H=\left\{H_{1} ; H_{2} ; H_{3} ; \cdots ; H_{m}\right\}$. 
f) Single-factor fuzzy evaluation to establish fuzzy matrix; Single-factor fuzzy evaluation is judged from one factor to determine the degree to which the object belongs to the set of judgment elements. One by one for each factor $U_{i}(i=1,2,3, \cdots, m)$ To quantify if the factor is concentrated on the $i^{\text {th }}$ factor $U_{i}$ To judge, it sets the $\mathrm{m}^{\text {th }}$ element for the judgment $V_{m}$ The degree of affiliation is $r_{j}$, Judgment results can be expressed as fuzzy sets $R_{i}=r_{i 1} / V_{1}+r_{i 2} / V_{2}+\cdots+r_{i j} / V_{m} ; \quad R_{i}=\left\{r_{i 1}, r_{i 2}, r_{i 3}, \cdots, r_{i m}\right\}$. By fuzzy collection $R_{i}$ The matrix $\mathrm{R}$ is fuzzy matrix.

g) fuzzy comprehensive evaluation; comprehensive evaluation of single factor can only reflect the impact of a certain factor on the object of evaluation, but in fact all factors need to be comprehensively considered the comprehensive impact on the object of judgment. Therefore, multi-factor fuzzy comprehensive evaluation results are needed: $B=A \times R$, B For fuzzy evaluation set, $b_{j}(j=1,2,3, \cdots, m)$ As a fuzzy comprehensive index.

\section{EVAluation OF ZHOUSHAN MARITIME SAR CAPABILITIES}

Because of the complicated maritime search and rescue process, many influencing factors and complicated relations, the factors themselves have a lot of uncertainties. Therefore, combining with the actuality of search and rescue in Zhoushan sea, this paper uses the principle of fuzzy mathematics, combines the qualitative and quantitative analysis based on fuzzy inference, Inaccurate and uniformed method to analyze and evaluate the maritime search and rescue capability of Zhoushan sea area, reduce the subjectivity in the decisionmaking process and provide a reference for the maritime search and rescue in Zhoushan sea area.

\section{A. Evaluation Factor Set}

According to the principle of evaluation index and the analysis of influencing factors, this paper conducted a questionnaire survey, research and index screening on the search and rescue capability of the sea in Zhoushan sea area. With reference to the manual of maritime SAR, the primary index and the first grade index were determined. Table 1 is the questionnaire to obtain the factor set:

TABLE I. ZHOUSHAN MARITIME SAR CAPABILITIES ASSESSMENT EXPERT CONSULTATION FORM

\begin{tabular}{cccccc}
\hline $\begin{array}{c}\text { Evaluation } \\
\text { index }\end{array}$ & $\begin{array}{c}\text { The value } \\
\text { of the } \\
\text { evaluation } \\
\text { indicator }\end{array}$ & Distinction & Good & Average & Fair $\quad$ Poor \\
\hline physical & $a_{1}$ & $\checkmark$ & & \\
fitness & & & & \\
$\ldots$ & & & $\checkmark$ & \\
$\begin{array}{c}\text { Rescue } \\
\text { distance }\end{array}$ & & & &
\end{tabular}

According to the statistical results of expert questionnaire, the human factors, environmental factors, rescue factors and search and rescue communications factors have a greater impact on the search and rescue capabilities evaluation results, so the following set of risk factors are identified:

$U=\left\{\right.$ Human factor $U_{1}$, environmental factor $U_{2}$, Rescue factors $U_{3}$, Search and rescue communications factors $U_{4}$ \}, among them:

$U_{1}=$ \{Physical fitness $U_{11}$, Willpower $U_{12}$, Psychological quality $U_{13}$, Professional knowledge and skills $U_{14}$, Self-help measures $\left.U_{15}\right\}$;

$U_{2}=$ \{sea conditions $U_{21}$, visibility $U_{22}$, traffic flow $U_{23}$, Temperature $U_{24}$, Land distance $\left.U_{25}\right\}$;

$U_{3}=$ \{rescue distance $U_{31}$, Rescue ability $U_{32}$, Aid $U_{33}$, Rescue operation $\left.U_{34}\right\}$;

$U_{4}=$ \{Positioning accuracy $U_{41}$, False alarm rate $U_{42}$ Search and rescue communications priority $\left.U_{43}\right\}$;

\section{B. Factors to Determine the Level of Evaluation}

According to the actual situation in Zhoushan sea area to determine the various factors of rating, comment, scale scores. The evaluation level of the qualitative evaluation index of the research is determined by expert questionnaire method and research method, and the scale grade is divided into five levels as follows:

TABLE II. RATING SCALE

\begin{tabular}{ccccc}
\hline Distinction & Good & Average & Fair & Poor \\
0.9 & 0.7 & 0.6 & 0.3 & 0.1 \\
\hline
\end{tabular}

\section{Determination of Weights}

After determining the evaluation index, we continue to use the expert questionnaire for the selected index to determine the final evaluation index. According to the formula 1 can calculate the evaluation index score:

$$
r_{i j}=n_{i j} \cdot \sum_{j=1}^{5} n_{i j} \quad(i=(1,2,3, \cdots, m))
$$

In the formula $n_{i j}$ Said it will be the first i A rating of the first assessment $\mathrm{j}$ Level of experts, come to the statistical results in Table 3: 
TABLE III. ZHOUSHAN MARITIME SAR CAPABILITIES COMPREHENSIVE RATING VALUE

\begin{tabular}{|c|c|c|c|c|c|c|c|}
\hline \multirow{7}{*}{$\begin{array}{c}\text { Fuzzy } \\
\text { Comprehens } \\
\text { ive } \\
\text { Evaluation } \\
\text { of Maritime } \\
\text { SAR Ability }\end{array}$} & \multirow{2}{*}{ Subsystem } & \multirow{2}{*}{ Evaluation index } & \multirow{2}{*}{ Weights } & \multicolumn{4}{|c|}{ Fuzzy relationship } \\
\hline & & & & Better & Good & Average & Poor \\
\hline & \multirow{2}{*}{ Human factors $U_{1}=0.35$} & physical fitness $U_{11}$ & 0.200 & 0.15 & 0.30 & 0.35 & 0.20 \\
\hline & & $\ldots$ & & & & & \\
\hline & $\ldots$ & & & & & & \\
\hline & \multirow[b]{2}{*}{ Rescue factor $U_{3}=0.25$} & $\ldots$ & & & & & \\
\hline & & $\begin{array}{c}\text { Rescue } \\
\text { operation } U_{34}\end{array}$ & 0.250 & 0.25 & 0.30 & 0.35 & 0.10 \\
\hline
\end{tabular}

\section{Evaluation}

As can be seen from Table 3, Zhoushan maritime SAR capabilities include three levels of primary, primary and secondary ratings, quantitatively get the impact of various factors on the search and rescue capabilities of Zhoushan sea and the size of the search and rescue capabilities of Zhoushan, the following were evaluated:

1) Primary evaluation

$$
U_{11}=0.15 \times 0.9+0.30 \times 0.7+0.35 \times 0.6+0.20 \times 0.3=0.615 ;
$$

Same reason can ask $U_{12}, U_{13} \ldots U_{43}$ See Table 4 for details.

2) a level of evaluation; according to the formula, where $B$ is the result of multiple factors, $A$ is the weight vector, $R$ is the fuzzy matrix, $U$ is the multi-factor set.

$B_{i}=A_{i} \times R_{i}=\left\{\begin{array}{lllll}0.200 & 0.100 & 0.100 & 0.375 & 0.225\end{array}\right\} \times\left\{\begin{array}{cccc}0.15 & 0.30 & 0.35 & 0.20 \\ 0.10 & 0.35 & 0.40 & 0.15 \\ 0.20 & 0.35 & 0.25 & 0.20 \\ 0.20 & 0.25 & 0.35 & 0.20 \\ 0.20 & 0.30 & 0.35 & 0.15\end{array}\right\}$

$U_{1}=0.180 \times 0.9+0.291 \times 0.7+0.345 \times 0.6+0.184 \times 0.3=0.6279$, then $U_{1}$ the "human factor" evaluation result is medium. The same token can be evaluated $U_{2}, U_{3}, U_{4}$ See Table 4 for details.

3) secondary evaluation; according to a method of evaluation, available from the data:

$$
\begin{aligned}
& B=A \times R=\left(\begin{array}{llll}
0.35 & 0.25 & 0.25 & 0.15
\end{array}\right) \times\left\{\begin{array}{llll}
0.180 & 0.291 & 0.345 & 0.184 \\
0.300 & 0.325 & 0.250 & 0.125 \\
0.250 & 0.350 & 0.238 & 0.163 \\
0.405 & 0.225 & 0.185 & 0.185
\end{array}\right\} \\
& =\left(\begin{array}{llll}
0.261 & 0.304 & 0.270 & 0.164
\end{array}\right)
\end{aligned}
$$

$$
\begin{aligned}
& \mathrm{U}=0.261 \times 0.9+0.304 \times 0.7+0.270 \times 0.6+0.164 \times 0.3 \\
& \mathrm{U}=0.6589
\end{aligned}
$$

Zhoushan SAR capabilities assessment results for the good.

\section{E. Zhoushan Maritime SAR Capabilities Evaluation Results}

In order to quantitatively analyze the maritime SAR capability in Zhoushan sea area and to get the influence factors of SAR capacity in Zhoushan and its influence size and to better analyze its law.

TABLE IV. ZHOUSHAN MARITIME SAR CAPABILITIES EVALUATION RESULTS TABLE

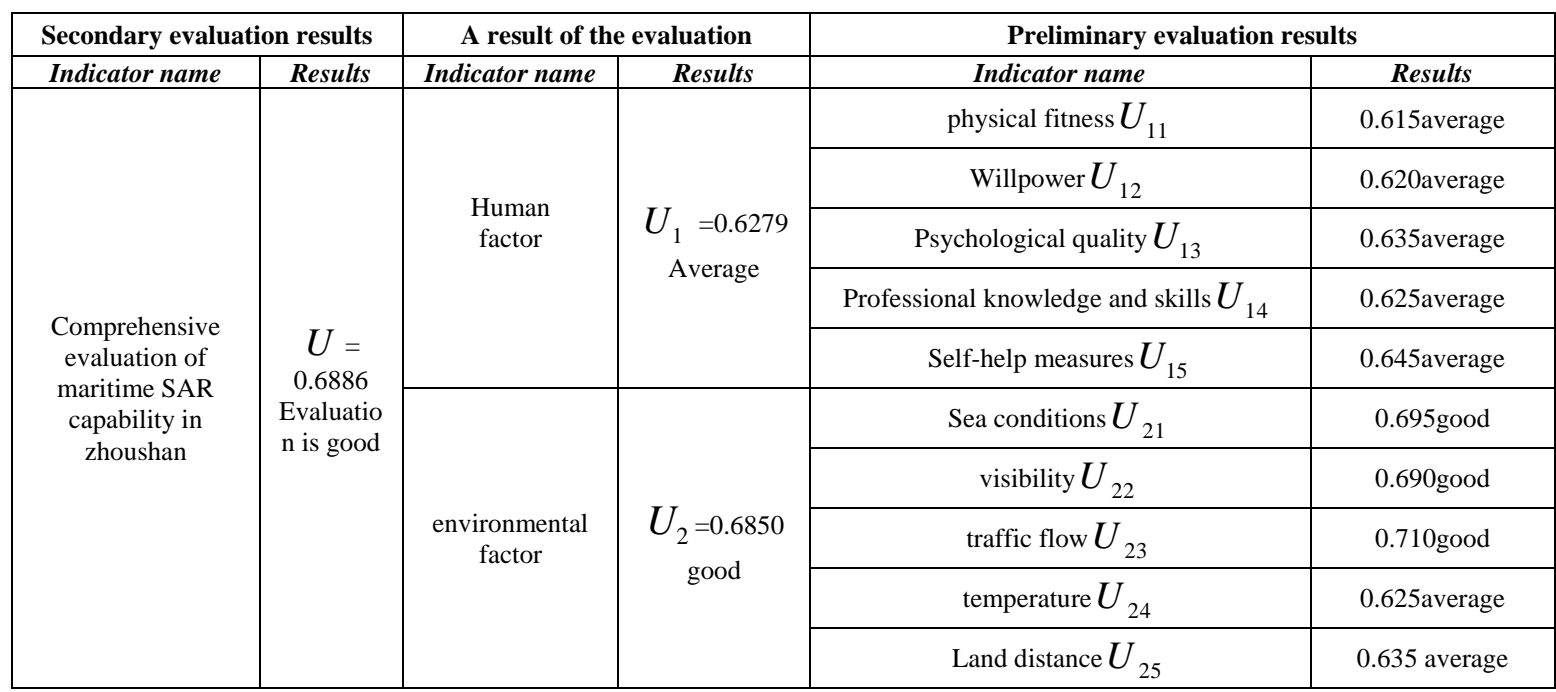




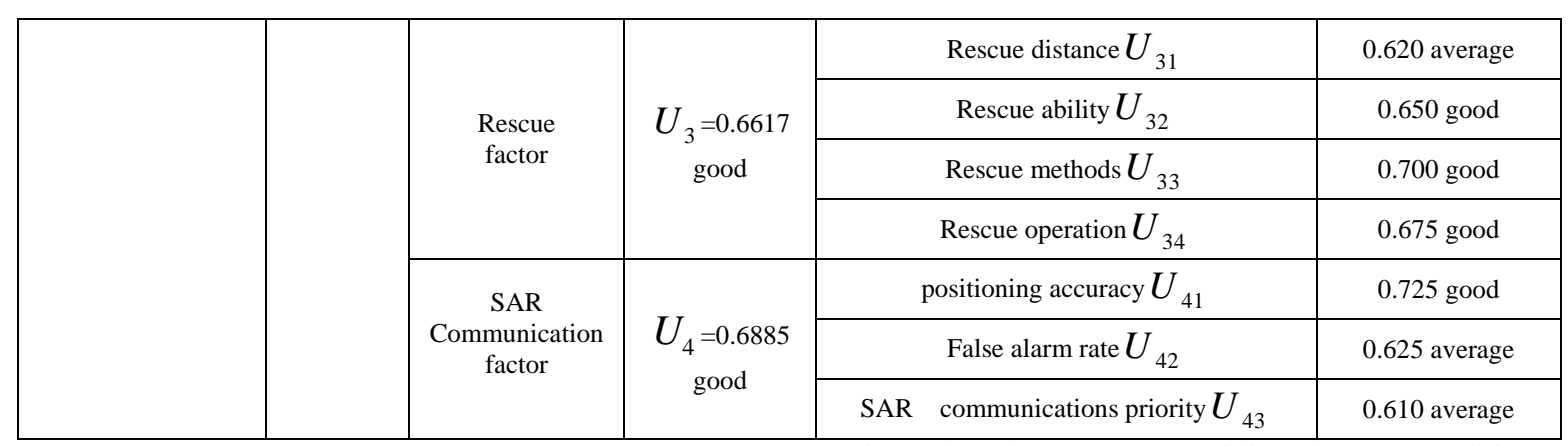

\section{F. Evaluation Results Analysis}

As can be seen from the evaluation results in Table 4, in general, the results of maritime search and rescue capability assessment in Zhoushan sea area $U=0.6589$, the rating is good.

Indicating that Zhoushan maritime search and rescue agencies can make a prompt response to the distress events and can complete the maritime search and rescue mission in the area according to the plan and can effectively organize and coordinate the completion of maritime search and rescue at all scales and ensure the man-made and environmental factors in Zhoushan maritime search and rescue process, Rescue factors and search and rescue communications and other influencing factors benign interaction, so as to maintain a high maritime search and rescue area success rate, according to Zhoushan Maritime Bureau annual report, the past five years, Zhoushan maritime search and rescue success rates are above $95 \%$.

According to an evaluation of the impact of factors can be seen, the human factor evaluation results $U=0.6279$, relatively low, is to determine the maritime search and rescue capabilities Zhoushan weak links. Although search and rescue personnel at sea search and rescue workers have good professional knowledge and professional skills, search and rescue workers in search of rescuers have a large number of fishing vessels and gravel boats in Zhoushan area. Their crew members' physical quality, willpower, psychological quality, professional knowledge and skills, and self-help Measures are relatively low, resulting in a comprehensive evaluation of indicators of human factors as a result, to be caused by the participants in the process of search and rescue Zhoushan sea attaches great importance to strengthen seafarers and search and rescue personnel training and determination of physical fitness to strengthen maritime search and rescue participants in the professional Skills and psychological knowledge training, and actively organize the Zhoushan maritime search and rescue drills to improve the running-in between professionals and sailors and strive to improve the professional qualities and skills of sea participants, which plays an important role in enhancing the search and rescue capability of Zhoushan sea areas.

According to the analysis of individual factors according to the result of primary evaluation, the results of the traffic flow $(\mathrm{U}=0.710)$ and the positioning accuracy $(\mathrm{U}=0.725)$ are higher because of the development of Ningbo-Zhoushan port in recent years, Zhoushan Fishing Ground is the largest fishing ground in China. There are also a large number of fishing boats in Zhoushan sea area, and the waterway covers almost the entire Zhoushan area, providing better conditions for the mutual rescuing of ships on the sea in Zhoushan. Coupled with the relevant government departments to effectively manage them, standardize the merchant vessels, fishing routes, while strengthening the ship's advanced technology and equipment applications, for maritime search and rescue play a positive role. In contrast, the search and rescue communications priority $(\mathrm{U}=0.610)$, false alarm rate $(\mathrm{U}=$ $0.625)$, rescue distance $(U=0.620)$ and other factors lower the evaluation results show that the Zhoushan marine communications security needs to be improved, especially the staff For pass-through device operation. The main reason is that there are many fishing vessels and ore vessels navigating in the waters of Zhoushan. The quality of personnel on board ships is low, and the operation of GMDSS equipment is not familiar enough with poor English skills. Therefore, there are two ways to solve the current situation: First, strong personnel training to improve skills. At present, the Ministry of Agriculture has made compulsory requirements for oceangoing fishing boats' certification of radio operators. A large number of fishing vessel senior officers all return to learn the basic principles and operations of ship-borne radio communication equipment. Second, Intensified supervision and requires joint enforcement by maritime authorities and fishery authorities, Resolutely banned the ship for undocumented ship, according to the law for undocumented crew. For the salvage distance indicator is too low, should continue to update and introduce advanced search and rescue equipment, expand the search and rescue distance, while the rational distribution of professional search and rescue forces site and standby point, planning professional search and rescue power jurisdiction, improve the professional search and rescue power complementarity mechanism to ensure that areas of expertise Full coverage of the power area and climate provides a solid guarantee for the improvement of the search and rescue capability of the sea in Zhoushan.

\section{CONCLUSION}

In this paper, a comprehensive evaluation model of maritime SAR capability in Zhoushan sea area is established by using the fuzzy comprehensive evaluation method. The ability of maritime SAR at Zhoushan sea area and its influencing factors are quantitatively analyzed. Based on the results, the causes and countermeasures are analyzed, providing reference for effectively improving the search and rescue capability of Zhoushan sea. 


\section{ACKNOWLEDGMENT}

This paper was supported by Zhejiang University Student Science and Technology Innovation Program and New Miao Talent Program, No. 2016R411023.

\section{REFERENCES}

[1] Xu Zhiyuan. National maritime search and rescue capabilities assessment and countermeasures [D]. Dalian: Dalian Maritime University .2007.

[2] Ren Zhijun. Application of Fuzzy Comprehensive Evaluation Method in Positioning of Power Lines in Loess Area [J] .Journal of Engineering Investigation.2006.

[3] Xu Wenmei. China's maritime search and rescue status and recommendations [J]. Water Transport Management .2009.

[4] FAN Xi-wei. Research on maritime search and rescue environment [D]. Dalian: Dalian Maritime University .2013. 